\title{
Efficient Role Assignment Scheme for Multichannel Wireless Mesh Networks
}

\author{
Sunmyeng Kim \\ Department of Computer Software Engineering \\ Kumoh National Institute of Technology \\ Gumi, South Korea
}

\author{
Hyun Ah Lee \\ Department of Computer Software Engineering \\ Kumoh National Institute of Technology \\ Gumi, South Korea
}

\begin{abstract}
WMN) is cost-effective access network architecture. The performance of multi-hop communication quickly reduces as the number of hops becomes larger. Nassiri et al. proposed a Molecular MAC protocol for autonomic assignment and use of multiple channels to improve network performance. In the Molecular MAC protocol, each node forms a shortest path-spanning tree to a gateway node linked to a wired Internet. After a tree is formed, the nodes with an even-numbered depth and an odd-numbered depth are assigned with the roles of a nucleus and an electron, respectively. After such roles are assigned, each nucleus selects an idle channel. However, this protocol has the following drawback; since the nodes with an even-numbered depth are assigned with the role of a nucleus, there are many nuclei in the topology. The number of assigned channels tends to increase, since each nucleus selects an idle channel that is not currently being occupied by its neighboring nuclei. In wireless communications networks, channels are very important resources. Thus, it is necessary to assign the minimum number of channels as little as possible. To do so, this paper proposes an efficient role assignment scheme, which can reduce the number of assigned channels by reducing the number of nodes assigned as nuclei and preventing nodes within the transmission range of each other from becoming nuclei. Based on various simulation results, the proposed scheme was verified.
\end{abstract}

\section{Keywords—role assignment; multichannel; mesh network}

\section{INTRODUCTION}

A wireless mesh network (WMN) is a cost-effective access network architecture. It is a promising wireless technology for numerous applications. It is gaining significant attention as a possible way for Internet service providers (ISPs) and carriers in order to provide wireless broadband service. In WMNs, nodes are consisted of mesh routers and mesh clients [1]. Mesh routers have minimal mobility and form the backbone of WMNs. When a node is within the transmission range of another node, they are considered as neighbors, and there is a wireless link between them. Some nodes, called gateways, are connected to the wired network, which connects the WMNs to rest of the Internet.

The packets sent by end users travel through wireless mesh networks over multiple hops. And gateway nodes relay traffic to and from the wired Internet. The performance of multi-hop communication quickly reduces as the number of hops becomes larger due to intra-flow and inter-flow interferences [2-4]. This is because a wireless link is shared among neighboring nodes.

With a single channel, nodes operate on the same channel. Therefore, network performance decreases. Nodes can be equipped with multiple radios and channels. This means there is a unique frequency used for each wireless hop, and thus enables separation of wireless collision domain and reduces the interference and contention. This can significantly improve network performance without bandwidth degradation.

The design of the MAC protocol is the most likely challenge in WMNs. An interesting problem in WMNs is how to efficiently utilize multiple channels. Several MAC protocols for handling multiple channels have been proposed in the literature [5-17].

The algorithms proposed in [5] select channels for the mesh radios to minimize interference within the mesh network and between the mesh network and co-located wireless networks. A new method for the interference estimation is suggested. All available channels are periodically monitored by each mesh node and measured information of internal channel usage and external interference is shared with mesh nodes within interference range. Duarte et al. use game theory to design a systematic approach to utilize partially overlapped channels in WMNs while minimizing the adverse effect of adjacent channel interference [6]. In [7], both centralized and distributed algorithms are presented, which aim to minimize the number of pairs of links that are interfering. The Molecular MAC protocol was proposed to organize the mesh network according to the molecular analogy [8-12]. It divides the network into atoms with nucleus nodes operating on fixed channels and electrons that dynamically switch channels between neighbor nuclei. Electrons can be shared by numerous atoms.

In the Molecular MAC protocol, each node forms a shortest path-spanning tree to a gateway node linked to a wired Internet. After a tree is formed, the nodes with an even-numbered depth and an odd-numbered depth are assigned with the roles of a nucleus and an electron, respectively. After such roles are assigned, each nucleus selects an idle channel that is not currently being occupied by its neighboring atoms with the assistance from electrons in the same atom.

Since a node with an even-numbered depth is a nucleus in a spanning tree, the Molecular MAC protocol can support many nuclei, in which nodes within the transmission range of each other can be nuclei. The number of assigned channels in the Molecular MAC protocol tends to increase, since each nucleus

This research was supported by Basic Science Research Program through the National Research Foundation of Korea (NRF) funded by the Ministry of Education, Science and Technology (2010-0025495). 
selects an idle channel that is not currently being occupied by its neighboring nuclei. In wireless communications networks, channels are very important resources. Thus, it is necessary to assign the minimum number of channels as little as possible. To do so, this paper proposes an efficient role assignment scheme, which can reduce the number of assigned channels by reducing the number of nodes assigned as nuclei and preventing nodes within the transmission range of each other from becoming nuclei. The proposed scheme adopts the basic operating principles of the Molecular MAC protocol, though the role assignment scheme is revised. For role assignment, the Molecular MAC protocol uses the shortest path-spanning tree, but the proposed scheme introduces a new metric by considering three components affecting network performance, and assigns the role of each node based on it. After the role assignment in the proposed scheme, nodes assigned as nuclei select channels with the help of electrons as in the Molecular MAC protocol.

The paper is organized as follows. We give a brief introduction of the Molecular MAC protocol and its role assignment problem in Section II. In Section III, the proposed scheme is presented in detail. In Section IV, performance studies are carried out through simulation results. Finally, we draw a conclusion in Section V.

\section{RELATED WORK}

This Section is designed to briefly specify the Molecular MAC protocol and then touch on certain problem relating to the role assignment.

\section{A. Molecular MAC Protocol}

The IEEE 802.11 wireless network functions well in the infrastructure mode. In addition, it can provide a fair bandwidth to all users by slightly modifying a channel access method. Nevertheless, the IEEE 802.11 network may incur numerous problems on a multi-hop network. The Molecular MAC protocol expands the IEEE 802.11 network in order to transmit data packets on the multi-hop network effectively.

Since the IEEE 802.11 access method works well on a single-hop network, the Molecular MAC protocol divides a wireless mesh network into different spatially distributed atoms. Each atom uses a channel not used by other neighboring atoms. An atom is composed of one nucleus and several electrons, and a nucleus selects a channel to be used by its own atom. Any node within an atom's boundary plays a role as an electron and belongs to a neighboring atom. An electron directly communicates with its nuclei; however, since there is no direct link between electrons, a direct communication among them cannot be implemented. In addition, due to no direct link among nuclei, direct communications among them are also not possible. Therefore, the communication among neighboring electrons can be handled by nuclei while the communication among nuclei can be executed by neighboring electrons.

In the Molecular MAC protocol, each node is assigned with the role as a nucleus or an electron while each nucleus selects a channel to be used by its own atom. Accordingly, each node forms a shortest path-spanning tree to a gateway node linked to a wired Internet. After a tree is formed, the nodes with an even- numbered depth and an odd-numbered depth are assigned with the roles of a nucleus and an electron, respectively. After such roles are assigned, each nucleus requests its own electrons for channel information. Channel information includes a list of active channels and their activities. A list of active channels includes the numbers of active channels in the corresponding electrons' parent atoms. A channel activity is a parameter that is designed to indicate how many data packets are transmitted on each channel in the active channel list, which is expressed in the number of packets transmitted. Requested by a nucleus, each electron makes up a list of active channels, measures each channel's activities, and accordingly responds to the nuclei. Then, a nucleus receives certain responses from all neighboring electrons and accordingly selects a channel according to a subsequent rule. 1) After a list of active channels is received from all neighboring electrons, a channel is randomly chosen out of those non-active channels. 2) If every channel is currently occupied, a channel with the least activity is selected. Once a nucleus allocates a channel, its neighboring electrons use the channel. The corresponding electrons use all channels allocated by their atoms' nuclei.

Fig. 1 illustrates a fundamental structure of the Molecular MAC protocol. As shown in the figure, there are 2 atoms, 2 nuclei and 6 electrons. The atom 1 includes the nucleus $\mathrm{N} 1$ and the electrons (E1, E2, E3 and E4), and uses the channel 1. The atom 2 includes the nucleus $\mathrm{N} 2$ and the electrons (E3, E4, E5 and E6), and uses the channel 2. The electrons E3 and E4, shared by the two atoms, use both channel 1 and channel 2. If a neighboring atom's nucleus requests information for the allocation of a channel, the corresponding electrons transmit their channel activity information. Furthermore, the electrons E1/E2, E3/E4 and E5/E6 also transmit the active channel lists including the channel 1 , the channels $1 \& 2$ and the channel 2, respectively, to the nucleus. Once all channels are allocated, communications are processed as follows: As shown in the figure, when certain data are to be transmitted from E1 to E6, E1 transmits the data to N1 using the channel 1 while N1 transmits the date back to E3 or E4. Then, E3 or E4 transmits the data to $\mathrm{N} 2$ using the channel 2 while $\mathrm{N} 2$ transmits the data back to E6. The neighboring electrons, E1 and E2, do not directly communicate with each other but communicate via the nucleus N1.

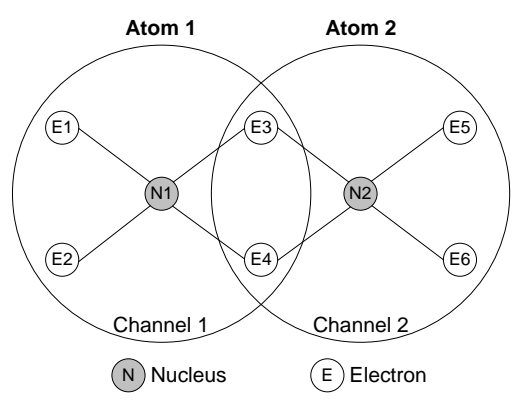

Fig. 1. Basic architecture of the Molecular MAC protocol

\section{B. Role Assignment Problem}

There is a problem in role assignment scheme of the Molecular MAC protocol. First of all, each node forms a shortest path-spanning tree to a gateway node connected to the 
wired Internet. After forming the tree, a node of which depth is an even number is assigned as a nucleus, and a node of which depth is an odd number as an electron. Since this scheme prefers the shortest path to the gateway node, the number of nuclei tends to increase, and that of assigned channels also increases. In addition, it only takes the even-numbered depth into account in role assignment. Nodes within the transmission range of each other can become nuclei, so the channel reuse ratio is low. Consequently, the number of assigned channels is increased.

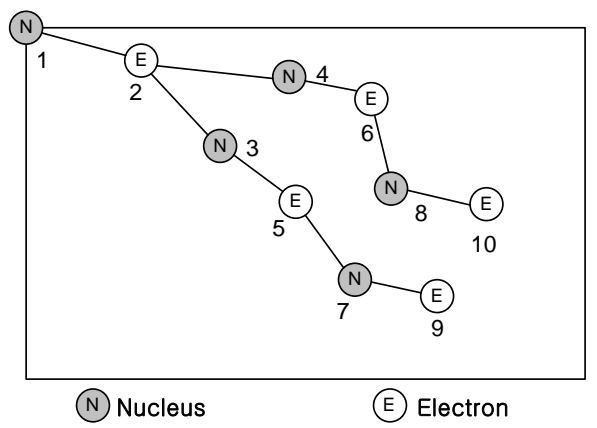

Fig. 2. Role assignment problem

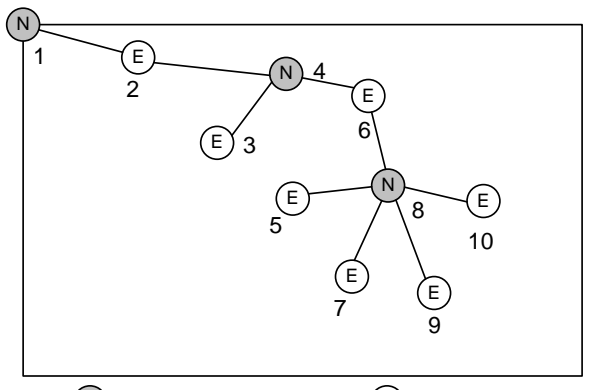

(N) Nucleus (E) Electron

Fig. 3. Example of role assignment with the proposed scheme

Fig. 2 shows a problem of role assignment in the Molecular MAC protocol. There are 10 nodes in the figure, and node 1 is a gateway node connected to the wired Internet. Remaining nodes form a shortest path-spanning tree from node 1 and are assigned roles. Since the depth of nodes 3, 4, 7 and 8 is an even number, the role of a nucleus is assigned to each of these nodes. As the depth of remaining nodes is an odd number, the role of an electron is assigned to each of them. As shown in the figure, nodes 3 and 4, and nodes 7 and 8 are assigned as nuclei, even though they are located within the transmission range of each other. Thus, this scheme causes the waste of channels.

To solve this problem, this paper introduces a new metric, and proposes a new scheme of assigning roles to nodes. With this scheme, it is possible to prevent nodes located within the transmission range of each other from becoming nuclei, and allow the number of electrons supported by a nucleus approach the number which optimizes the network performance. Fig. 3 shows an example of role assignment with the proposed scheme. It shows that the number of nuclei is reduced from 5 to 3 compare to Fig. 2. Accordingly, the number of assigned channels is also reduced. More details are described in Section III.

\section{Proposed Role Assignment Scheme}

In this paper, we propose a new scheme to solve the problem of assigning roles to nodes in the Molecular MAC protocol. The proposed scheme adopts the basic operating principle of the Molecular MAC protocol without any modifications, though some parts related to the role assignment of nodes are modified. In the proposed scheme, a node which fully meets the following conditions is assigned as a nucleus: that is, a node with the optimal number of electrons and a node which can send data of electrons linked to it to a gateway node in a timely way. And then, to avoid selecting nodes within the transmission range of each other as nuclei, all nodes located within the transmission range of the node selected as a nucleus are assigned as electrons. To do that, we introduce a new metric, which is called as Expected Transmission Performance (ETP).

\section{A. Overall Flow of Tree-Forming Process}

In the proposed scheme, a node forms a tree to a gateway node by using the ETP metric. Each node is assigned one of the following roles: nucleus, electron, unassigned, or candidate nucleus. When the role assignment is completed, every node is assigned the role of a nucleus or an electron. An unassigned node is a node to which the role of nucleus or electron is not assigned in the tree-forming process. A candidate nucleus means a node, which has potential of being a nucleus among unassigned nodes in the role assignment process. Thus, it is also an unassigned node. In the first stage of making a tree, the role of every node is unassigned. A gateway node is assigned as a nucleus in default. The tree-forming process is as follows:

1) Every unassigned node within the transmission range of the gateway node is assigned as an electron, and forms a tree to connect to the gateway node.

2) Each electron node assigns every unassigned node within its transmission range as a candidate nucleus node.

3) For candidate nucleus nodes, ETP metric table as shown in Table I is made.

TABLE I. ETP METRIC TABLE

\begin{tabular}{|c|c|}
\hline Electron ID & Metric Value \\
\hline & \\
\hline & \\
\hline
\end{tabular}

In the table, an electron ID is the identity of an electron which assigns a node as a candidate nucleus node in Step 2. How to calculate metric values is described in subsection III.B in detail.

4) After making an ETP metric table for all candidate nucleus nodes, a candidate nucleus node with the largest metric value is assigned as a nucleus.

5) A nucleus node newly assigned in Step 4 assigns every unassigned node within its transmission range as an electron and forms a tree by connecting them to itself.

6) The newly assigned nucleus node forms a tree by connecting to a node with electron ID of the largest metric value in Table I.

7) It resets every candidate nucleus node to unassigned status, and initializes its ETP metric table. 
8) Until the role of nucleus or electron is assigned to all unassigned nodes, Step 2 to 7 is repeated.

\section{B. Calculation of ETP Metric Value}

As a nucleus, the proposed scheme selects a node that has optimal number of electrons and sends data of electrons to the gateway node at the maximum data rate. For this end, the following three conditions shall be met to apply the proposed scheme. First, a nucleus shall have optimal number of electrons, because the network performance is deteriorated if the number of electrons connected to the nucleus is too many or too few. Second, a nucleus shall be able to send data at high data rate to connected electrons. Finally, data transmission time from the nucleus to the gateway node shall be short. To satisfy the above conditions, the proposed ETP metric is made of the following three components: $N O_{\text {Node }}, D A T A_{\text {Rate }}$, and $T X_{\text {Time }}$. Each metric component has a value of $[0,1]$, where ' 1 ' is the best and ' 0 ' is the worst. Thus, a candidate nucleus node with the largest ETP metric value is selected as a nucleus.

ETP metric component $N O_{\text {Node }}$ is obtained as the number of unassigned nodes within the transmission range of a candidate nucleus node. Wireless mesh networks can be implemented with various wireless technology including 802.11, 802.15, 802.16, cellular technologies or combinations of more than one type. With the WLAN popularizing, IEEE 802.11 MAC protocol has been adopted as the de-facto medium access control of wireless mesh networks. In IEEE 802.11 MAC protocol, network performance is enhanced following the growth of the number of nodes with access points, but the performance is deteriorated if the number exceeds a certain value. Thus, the number of nodes, which shows the maximum network performance, is defined as the optimal number of nodes. When the number of unassigned nodes within the transmission range of a candidate nucleus node approaches the optimal number of nodes, $N O_{\text {Node }}$ approaches 1 . On the contrary, if there is a large discrepancy with the optimal number of nodes, then it approaches ' 0 '. $N O_{\text {Node }}$ is calculated as follows:

$$
N O_{\text {Node }}=1-\left|\frac{O p t_{N}-n}{O p t_{N}}\right|
$$

where, $n$ is the number of unassigned nodes within the transmission range of a candidate nucleus node, and $\mathrm{Opt}_{N}$ is the optimal number of nodes. $|x|$ is the absolute value of $x$. When $n$ is two times or more than $O p t_{N}$, this metric component may have a negative value. To avoid this, $n$ is set to $2 \cdot O p t_{N}$.

When a candidate nucleus node is assigned as a nucleus, ETP metric component $D A T A_{\text {Rate }}$ is calculated by considering data rates with connecting electrons. The candidate nucleus node measures the distances to unassigned nodes within its transmission range and checks the data rate with each node with the data rate table based on the measured distances. Table II shows the relationship between the rate and the distance. This table is from a reference [18]. Although the reference showed the rate based on distance between two nodes, this paper is expressed the distance between two nodes after normalizing it. That is, a normalized distance is a value dividing the distance between two nodes by the transmission range of a node. Thus, it is expressed as $[0,1]$.

TABlE II. Data Rate According To The Distance Between Two NODES IN OPEN ENVIRONMENT IN $802.11 \mathrm{~B} / \mathrm{G}$

\begin{tabular}{|c|c|}
\hline Normalized Distance & Data Rate(Mbps) \\
\hline 0.125 & 54 \\
\hline 0.213 & 36 \\
\hline 0.300 & 18 \\
\hline 0.498 & 11 \\
\hline 0.649 & 6 \\
\hline 1.000 & 1 \\
\hline
\end{tabular}

With Table II, data rates between the candidate nucleus node and all unassigned node within its transmission range are checked and average data rate $\left(A v g_{\text {rate }}\right)$ is calculated as follows:

$$
A v g_{\text {rate }}=\sum_{i=1}^{n} \text { Rate }_{i} / n
$$

where, $n$ is the number of unassigned nodes within the transmission range of a candidate nucleus node, and Rate $_{i}$ is the data rate between the candidate nucleus node and an unassigned node $i$.

ETP metric component $D A T A_{\text {Rate }}$ is calculated by dividing average data rate by the maximum rate $\left(M A X_{\text {rate }}\right)$ supported by the network as follows. For example, the maximum rate in Table II is 54 Mbps.

$$
D A T A_{\text {Rate }}=\frac{A v g_{\text {rate }}}{M A X_{\text {rate }}}
$$

Let us define a gateway transmission time and neighbor transmission time as the length of time required to send a data packet from a node to the gateway node and between neighbor nodes, respectively.

ETP metric component $T X_{\text {Time }}$ is obtained by using the gateway transmission time and neighbor transmission time.

In Step 5 in subsection III.A, after assigning a candidate nucleus node as a nucleus, the nucleus node assigns unassigned nodes within its transmission range as its electrons, and then connects as a tree. At this time, the nucleus node sends the gateway transmission time to each electron node. In addition, each electron node adds the gateway transmission time received from its nucleus and the neighbor transmission time between itself and its nucleus. In Step 2 in subsection III.A, it sends the sum to the candidate nucleus node.

When forming a tree with unassigned nodes, each node sends the unassigned nodes the gateway transmission time from itself to the gateway node. When receiving the gateway 
transmission time, an unassigned node sends another unassigned node the sum of the received gateway transmission time and the neighbor transmission time from itself to the previous node.

Let us denote data rate and neighbor transmission time of two neighboring nodes $i$ and $j$ as Rate $_{i, j}$ and TxTime $e_{i, j}$. The relationship between the two is expressed as follows:

$$
\text { TxTime }_{i, j}=\frac{1}{\text { Rate }_{i, j}}
$$

Now, let us denote TxTime $_{n \rightarrow 1}$ the gateway transmission time from an arbitrary node $n$ to the gateway node 1 . The gateway transmission time is expressed as the sum of neighbor transmission times between neighboring nodes, through which data is passed when sending a data packet from a node to the gateway node through the formed tree, as shown in the following expression:

$$
\begin{aligned}
\text { TxTime }_{n \rightarrow 1} & =\text { TxTime }_{n-1 \rightarrow 1}+\text { TxTime }_{n, n-1} \\
& =\sum_{i=n}^{2} \text { TxTime }_{i, i-1}
\end{aligned}
$$

where, $n$ is an arbitrary node and 1 is the gateway node. $n-1, n$ $2, \ldots$, and 2 are nodes placed on a path from the arbitrary node to the gateway node in the tree.

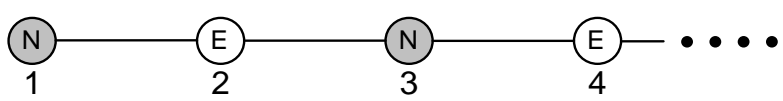

Fig. 4. Example of calculating TxTime Til $_{n \rightarrow 1}$

Fig. 4 shows an example of the process of calculating TxTime $_{n \rightarrow 1}$. There are 4 nodes in the figure, and node 1 is the gateway node. The gateway node is assigned as a nucleus, which assigns node 2 located within its transmission range as an electron and connects to the tree. And then it sends the gateway transmission time of TxTime $_{1 \rightarrow 1}(=0)$ to node 2. Node 2 assigns node 3 within its transmission range as a candidate nucleus node, and calculates the gateway transmission time ( Tx $_{\text {Time }_{2 \rightarrow 1}}$ ) from itself to the gateway node 1 and sends the result to node 3. TxTime $_{2 \rightarrow 1}$ is $0+$ TxTime $_{2,1}$. Node 3 is assigned as a nucleus, and then assigns node 4 within its transmission range as an electron and connects it as a tree. It then calculates the gateway transmission time (

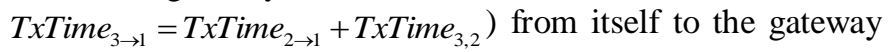
node 1 , and sends the result to node 4 . In addition, node 4 also calculates the gateway transmission time (

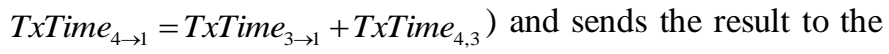
next node.
After every candidate nucleus node calculates the gateway transmission time, the largest value $\left(\right.$ TxTime $\left._{\max }\right)$ is selected. $N$ is a set of candidate nucleus nodes.

$$
\text { TxTime }_{\max }=\max _{i \in N} \text { Tx }_{\text {Time }} \rightarrow \rightarrow
$$

ETP metric component $T X_{\text {Time }}$ of an arbitrary node $n$ is calculated by using TxTime $_{\max }$ as follows:

$$
T x_{\text {Time }}=1-\frac{\text { TxTime }_{n \rightarrow 1}}{\text { TxTime }_{\max }}
$$

After calculating three metric components $\mathrm{NO}_{\text {Node }}$, $D A T A_{\text {Rate }}$, and $T X_{\text {Time }}$, the ETP metric value $(E T P)$ is obtained as follows:

$$
E T P=\alpha \cdot N O_{\text {Node }}+\beta \cdot D A T A_{\text {Rate }}+\gamma \cdot T X_{\text {Time }}
$$

where, $\alpha, \beta$, and $\gamma$ are weighting factors for each metric component $(\alpha+\beta+\gamma=1)$. ETP has a value of $[0,1]$, where ' 1 ' is the best.

\section{Simulation Results}

In this Section, we discuss the simulation results of the proposed scheme. To study the performance of the proposed scheme, we have implemented it. We compare them to the results of the Molecular MAC protocol. We simulated an IEEE 802.11 network with transmission rates of $54 \mathrm{Mbps}$ for data packets and of $6 \mathrm{Mbps}$ for control packets such as RTS, CTS and ACK, respectively. $O p t_{N}$ is 5. $\alpha, \beta$, and $\gamma$ are set to 0.3 , 0.3 , and 0.4 respectively.

The spanning tree construction proceeds as follows. First, the network elects a gateway node, which is connected to the wired Internet. Then, the other nodes construct a spanning tree rooted at the gateway node. We place the gateway node on the top-left corner and randomly the other nodes in a simulation topology.

Main performance metrics of interest are the number of nuclei, the number of assigned channels, the number of electrons per nucleus and the number of nuclei per electron. The number of nuclei is the number of nodes assigned as nuclei in the simulation topology. The number of assigned channels is the number of channels assigned to nuclei in the simulation topology. $\mathrm{T}$

hat is, it is the number of channels required to serve all the nodes in the simulation topology. The number of electrons per nucleus is the average number of electrons connected to a nucleus. The number of nuclei per electron is the average number of nuclei connected to an electron. All simulation results are averaged over ten simulations. In the simulation result, the proposed scheme is expressed as ETP. 


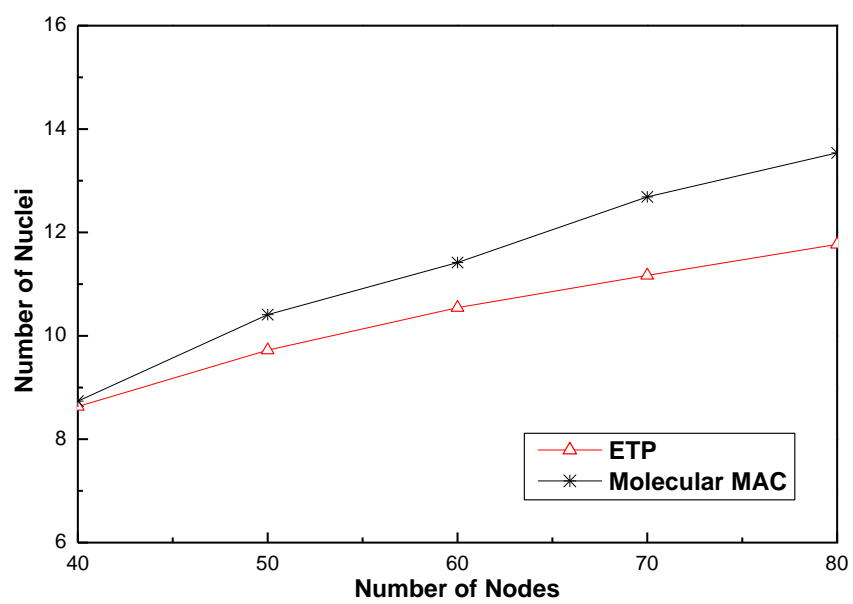

Fig. 5. Number of nuclei according to the number of nodes

Figs. 5, 6, 7, and 8 show change of network performance following the increase of the number of nodes used in the simulation topology. Fig. 5 shows that the number of nodes assigned as nuclei is increasing linearly in proportion to the number of nodes. When the number of nodes is 40 , about $22 \%$ of the nodes are nuclei in both schemes. As the number of nodes is increasing, the proportion is decreasing progressively. Thus, when the number of nodes is 80 , about $14.7 \%$ of the nodes are assigned as nuclei in the proposed scheme, while about $16.9 \%$ are in the Molecular MAC protocol. Fig. 5 shows that the number of nodes in the proposed ETP scheme is increasing slowly compared to that in the Molecular MAC protocol, and its proportion is also low.

Fig. 6 shows the number of channels assigned to serve all the nodes in the simulation topology. In the Molecular MAC protocol, the number of assigned channels is slowly increasing following the growth of the number of nodes. When the number of nodes is 40 , about 3.7 channels are assigned; when it is 80 nodes, 4.8 channels are assigned. However, in the proposed ETP scheme, it is maintained constantly regardless of the number of nodes. As explained in subsection II.B, in the Molecular MAC protocol, each node forms the shortest pathspanning tree to the gateway node, and a node of which depth is even numbered is assigned as a nucleus. Thus, as shown in Fig. 6, the number of nodes assigned as nuclei increases. In addition, only even-numbered depth is considered in role assignment, neighboring nodes within the transmission range of each other become nuclei and use different channels from each other, the channel reuse ratio is lowered. As a result, the number of assigned channels increases. However, the proposed ETP scheme takes the transmission range into account, only a node separated at an appropriate distance is assigned as a nucleus. Thus, there is no possibility that a neighboring node within the transmission range is a nucleus. If two nuclei are separated at a certain distance, then both of them operate without a hitch when they are using the same channel. Thus, the channel reuse ratio increases, and the number of assigned channels is also grown slowly.

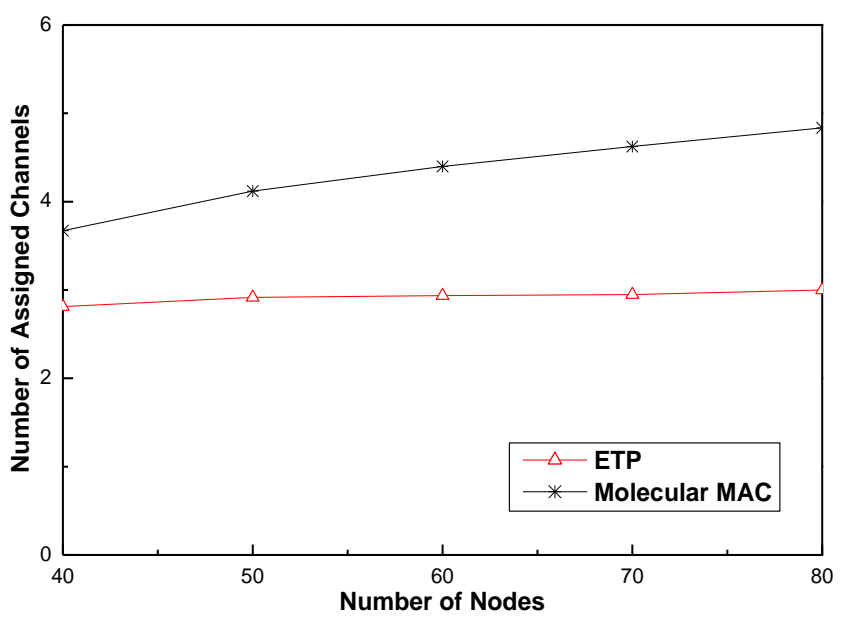

Fig. 6. Number of assigned channels according to the number of nodes

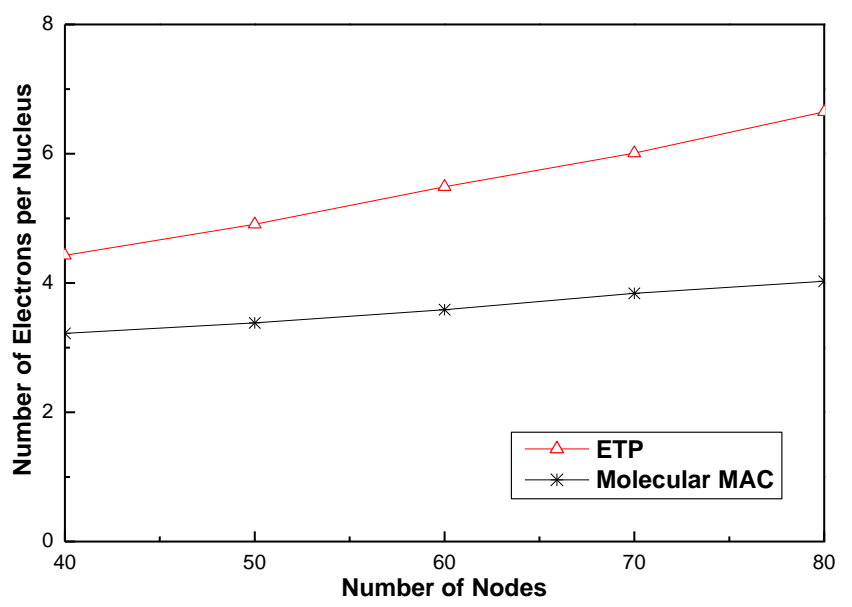

Fig. 7. Number of electrons per nucleus according to the number of nodes

Fig. 7 shows the number of electrons connected to each nucleus following the increase of nodes. As shown in Fig. 5, when the number of nodes is 40 , that of nuclei in the proposed ETP scheme is 8.6, while that of the Molecular MAC protocol is 8.7. And the number of electrons is 31.4 and 31.3, respectively. In average, the number of electrons connected to a nucleus can be obtained by dividing the number of electrons by that of nuclei. In theory, the average number of electrons is 3.7 $(=31.4 / 8.6)$ and $3.6(=31.3 / 8.7)$, respectively. However, Fig. 7 shows somewhat different results: 4.4 and 3.2, respectively. The cause of such discrepancy is as follows: As shown in Figs. 2 and 3, a leaf node in the tree graph is connected to a nucleus, though an electron in the middle is connected more than two nuclei. In this way, an electron in the middle is connected to several nuclei, so the number of electrons per nucleus counts some redundant nodes, which makes such discrepancy. As shown in Fig. 5, the number of nuclei is increasing more slowly in the proposed ETP scheme than in the Molecular MAC protocol; thus, the number of linked electrons is increasing relatively faster. 
Fig. 8 shows the number of nuclei connected to an electron following the increasing number of nodes. As shown in Figs. 2 and 3 , all the electrons are connected to more than one nucleus to communicate. Therefore, the result value shall be more than 1 in both schemes, though the Molecular MAC protocol shows a value of less than 1 . The cause is as follows: In the Molecular MAC protocol, a node with even-numbered depth is assigned as a nucleus. However, exceptionally, a leaf node is assigned as an electron not as a nucleus, even if it has even-numbered depth, and then connected to electrons.

That is, the last node with even-numbered depth does not communicate with the nucleus. When such nodes are increasing, there is intra-flow interference, which may deteriorate the network performance. The proposed ETP scheme follows the operating principle of the Molecular MAC protocol basically. However, since the proposed scheme minimizes such nodes, it is able to ameliorate the deterioration of network performance.

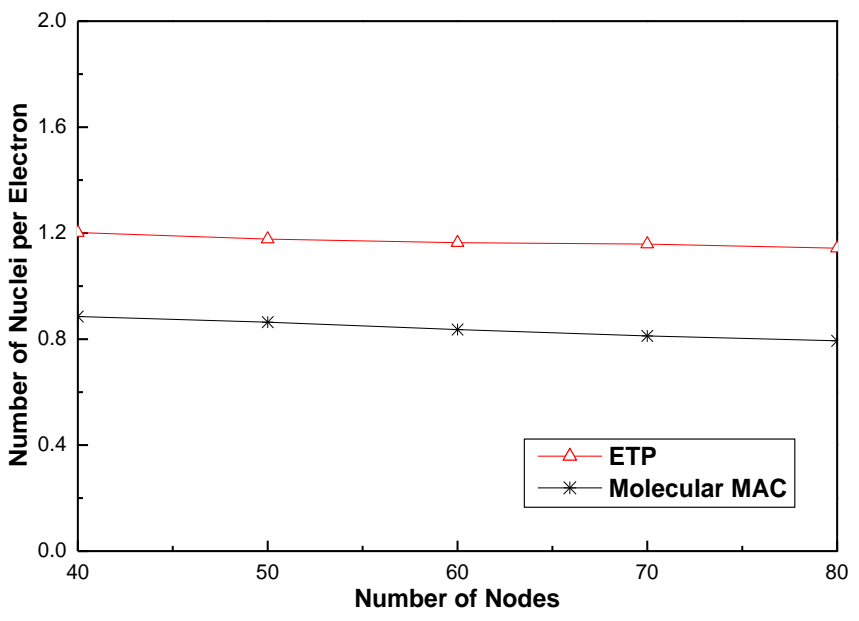

Fig. 8. Number of nuclei per electron according to the number of nodes

\section{CONCLUSION}

A wireless mesh network (WMN) is a promising wireless technology for numerous applications. The Molecular MAC protocol was proposed by adopting a molecular analogy. In the Molecular MAC protocol, the nodes with an even-numbered depth are assigned with the role of a nucleus, it supports many nuclei. Also, nodes within the transmission range of each other can become nuclei, so the channel reuse ratio is low. Consequently, the number of assigned channels increases. This paper proposes an efficient role assignment scheme, which can reduce the number of assigned channels by reducing the number of nodes assigned as nuclei and preventing nodes within the transmission range of each other from becoming nuclei.

The proposed scheme introduces a new metric by considering three components affecting network performance, and assigns the role of each node based on it. Simulation results show that the proposed scheme has better performance than the Molecular MAC protocol.

\section{REFERENCES}

[1] I.F. Akyildiza, X. Wang, and W. Wang, "Wireless mesh networks: a survey," Elsevier Computer Networks, vol. 47, no. 4, pp. 445-487, 2005.

[2] A. Iyer and C. Rosenberg, "Understanding the key performance issues with MAC protocols for multi-hop wireless networks," Wireless Communications and Mobile Computing, vol. 6, no. 6, pp. 745-760, 2006.

[3] H. Zhai, X. Chen, and Y. Fang, "Alleviating intra-flow and inter-flow contentions for reliable service in mobile ad hoc networks," in Proceedings of IEEE MILCOM, vol. 3, pp. 1640-1646, Nov. 2004.

[4] M. Karaliopoulos, T. Spyropoulos, and B. Plattner, "Interference-aware routing in wireless multihop networks," IEEE Transactions on Mobile Computing, vol. 10, no. 5, pp. 716-733, May 2011.

[5] Y. Kim, D. Jung, Y. Kim, S. Choi, and J. Ma, "Efficient interferenceaware channel allocation in multi-radio wireless mesh setworks," in Proceedings of International Conference on Advanced Communication Technology (ICACT 2012), pp. 920-925, 2012.

[6] P.B.F. Duarte, Z.M. Fadlullah, A.V. Vasilakos, and N. Kato, "On the partially overlapped channel assignment on wireless mesh network backbone: a game theoretic approach," IEEE Journal on Selected Areas in Communications, vol. 30, no. 1, pp. 119-127, Jan. 2012.

[7] A. Subramanian, H. Gupta, S. R. Das, and J. Cao, "Minimum interference channel assignment in multi-radio wireless mesh networks," IEEE Transactions on Mobile Computing, vol. 7, no. 12, pp. 1459-1473, Dec. 2008 .

[8] F. Theoleyre, B. Darties, and A. Duda, "Assignment of roles and channels for a multichannel MAC in wireless mesh networks," in Proceedings of 18th International Conference on Computer Communications and Networks (ICCCN 2009), pp. 1-6, Aug. 2009.

[9] B. Darties, F. Theoleyre, and A. Duda, "A Divide-and-conquer scheme for assigning roles in multi-channel wireless mesh networks," in Proceedings of IEEE 34th Conference on Local Computer Networks (LCN 2009), pp. 277-280, Zurich, Switzerland, Oct. 2009.

[10] D. Abdelali, F. Theoleyre, A. Bachir, and A. Duda, "Neighbor discovery with activity monitoring in multichannel wireless mesh networks," in Proceedings of IEEE Wireless Communications and Networking Conference (WCNC 2010), pp. 1-6, Apr. 2010.

[11] M. Nassiri, F. Theoleyre, M. Heusse, and A. Duda, "Molecular architecture for autonomic wireless mesh networks," In Proceedings of the ACM student workshop - CoNEXT 2007, Dec. 2007.

[12] F. Theoleyre, M. Heusse, and A. Duda, "Molecular MAC for multichannel wireless mesh networks," in Proceedings of IEEE 6th International Conference on Mobile Adhoc and Sensor Systems (MASS 2009), pp. 110-119, Oct. 2009.

[13] S. Pollak, V. Wieser, and A. Tkac, "A channel assignment algorithm for wireless mesh networks with interference minimization," in Proceedings of Wireless and Mobile Networking Conference (WMNC 2012), pp. 1721, 2012.

[14] K.N. Ramachandran, E.M. Belding, K.C. Almeroth, and M.M. Buddhikot, "Interference-aware channel assignment in multi-radio wireless mesh networks," in Proceedings of IEEE INFOCOM, pp. 1-12, Apr. 2006.

[15] K. Xing, X. Cheng, L. Ma, and Q. Liang, "Superimposed code based channel assignment in multi-radio multi-channel wireless mesh networks," in Proceedings of ACM MobiCom, pp. 15-26, 2007.

[16] H. Skalli, S. Ghosh, S. Das, L. Lenzini, and M. Conti, "Channel assignment strategies for multiradio wireless mesh networks: issues and solutions," IEEE Communications Magazine, vol. 45, no. 11, pp. 86-95, Nov. 2007.

[17] S. Avallone, G. Stasi, and A. Kassler, "A Traffic-aware channel and rate re-assignment algorithm for wireless mesh networks," IEEE Transactions on Mobile Computing, vol. 12, no. 7, pp. 1335-1348, Jul. 2013.

[18] Cisco Aironet 802.11a/b/g Wireless LAN Client Adapters (CB21 AG and P121AG) Installation and Configuration Guide, Appendix A, Technical Specification, http://www.cisco.com. 